\title{
Perceived Speed, Frustration and Enjoyment of Interactive and Passive Loading Scenarios in Virtual Reality
}

\author{
David Heidrich $^{1[0000-0002-8501-5089]}$, Annika Wohlan ${ }^{20000-0002-5635-2414]}$, and \\ Meike Schaller 2 [0000-0003-2378-0233] \\ 1 German Aerospace Center (DLR), Muenchener Straße, 82234 Weßling, Germany \\ ${ }^{2}$ German Aerospace Center (DLR), Linder Höhe, 51147 Cologne, Germany \\ \{david.heidrich, annika.wohlan, meike.schaller\}@dlr.de
}

\begin{abstract}
Long waits and disruptive loading breaks can evoke negative emotions, like frustration. While there is a lot of research on 2D-based loading scenarios, it is unclear how people react to loading screens in an immersive virtual reality (VR) environment. In this paper we conducted a user study to investigate the effects of interactive and passive loading screens on the users' loading screen experience (LSE) in VR. We measured perceived speed, enjoyment and frustration for long and short waiting times. Results show that interactive loading screens improved participants' LSE through increasing perceived speed and enjoyment, and decreased their frustration while waiting. Thus, previous findings of $2 \mathrm{D}$-based research were confirmed. Therefore, our research provides a first approach for further investigations of different loading screens in VR.
\end{abstract}

Keywords: Virtual Reality · Loading Screens · User Experience.

\section{Introduction}

Virtual reality (VR) technology is increasingly used in the context of data visualization $[20,14]$. Processing high amounts of data inside a VR-based real-time interactive system can introduce lag causing simulator sickness [19]. Hence, applications often reduce the complexity of the scene during processing by switching to a simple loading scenario, i.e., resulting in loading times. However, these disruptive loading breaks are undesirable for users as it can evoke negative emotions, like frustration $[4,6]$ and may therefore have a negative effect on the users' loading screen experience (LSE). We define LSE as the users' experience in a waiting situation while they receive feedback via a loading screen. The basic aspects of LSE are the perceived loading speed, the enjoyment, and the frustration caused by the perceived waiting time and the loading screen.

Since the intensity of emotions can be increased by a VR-based representation $[17,18]$, loading screens in VR could be perceived as even more negative or positive compared to 2D-based loading screens. Additionaly, users cannot avoid 
the loading scenario in a virtual environment by simply turning away from the screen, thus avoiding the loading situation. This could intensify a negative LSE even more.

In the context of 2D-based loading screens, passive loading scenarios (e.g., animations or progress bars) have a bigger negative impact on the user experience than interactive loading screens $[7,24]$. However, this has not yet been reviewed in a VR-based context. So, it is unclear whether existing literature on 2D-based loading scenarios is applicable to a VR-based context. Interestingly popular VRbased applications, like The Lab [23], use passive loading screens. In order to clarify the influence of interactive and passive loading scenarios on LSE, we tested an interactive and a passive loading screen in a VR-based application.

Contribution This article reports new findings on loading screens in VR. Thus, our contribution is twofold. 1) In a user study, differences in LSE between interactive and passive loading screens are measured by comparing an interactive and a passive loading scenario regarding perceived speed, frustration and enjoyment. 2) In the same user study, the influence of waiting duration (short and long) on perceived speed, frustration and enjoyment in interactive and passive loading scenarios is measured. The study results indicate a significant difference between interactive and passive loading screens in VR for both, short and long waiting times. Thus, the findings of 2D-based research are confirmed, i.e., interactive loading screens were better suited than passive loading scenarios. While this indicates that interactive loading screens might be generally better suited for VR-based applications, further research on the influence of immersion on the user experience during loading scenarios is needed.

\section{Related Work}

Forced breaks, i.e., loading screens, can have a negative impact on the user experience of an application. Users seem to dislike them that much, that in freeto-play video games, like Candy Crush, forced breaks are intentionally placed, resulting in users to voluntarily pay money to quit them [2]. However, in many applications loading screens cannot be avoided, e.g., when loading a big virtual environment. Visual Feedback on the loading progress while forced breaks has proven to be very helpful to avoid frustration [3] and to increase the users' tolerance regarding waiting times [15]. Additionally, latest research is proposing entertaining loading screen, e.g., interactive animations, as an even better solution than just visual feedback [7]. Interactive loading scenarios are perceived as faster and more enjoyable than a simple progress bar or non-interactive animation [7].

\subsection{Perceived Waiting Time}

Since the objective waiting time is mentally transformed into users' perceived waiting time, long waits can lead to a negative waiting experience [1]. In general, 
users tend to judge shorter waiting times to be more positive than longer ones [12]. Li and Chen [13] pointed out the importance of visual feedback design that has an influence on users' wait evaluations. For example, cartoon bars can improve users' wait experiences for short waiting times. Moreover, it has been found that the animation's speed is positively influencing users wait evaluations. Hence, with a faster animation, perceived waiting time is perceived shorter and users get more satisfied [22].

Kim et al. found that duration and the progress function affect the viewers' waiting time perception rather than the design of the loading symbol [11]. Hui and Tse [8] compared short, intermediate and long waiting times either with or without waiting-duration information. They found that waiting-duration information in longer waits results in a longer perceived waiting time, but increases the users' satisfaction while waiting. Contrary, Zhao et al. found that animation can also have a negative effect on user satisfaction during the wait for application loading. In their study they found that animated loading screens decrease users' satisfaction by affecting their duration estimation, thus creating an illusion of longer wait [24].

Nielsen [16] indicated that for waiting times longer than 10 seconds, users can not keep their attention on the dialog and want to perform other tasks. To that, Hurter et. al. [9] indicated that users desire to conduct extra activities than just waiting passively while waiting for a longer time. Hence, it is necessary to look more into long waits.

\subsection{Immersive VR}

The use of immersive VR technology, i.e., wearing a head-mounted display, is characterized by a high immersion. Immersion is achieved with objective system properties replacing sensory inputs from the real world with digital information [21]. That way, the users are disconnected from the real world. Since people tend to look for secondary tasks after a certain waiting duration [16], high immersion prevents the users from interacting with the real world. This is especially crucial in VR-based passive loading scenarios, where the only possible interaction is moving the hands around. Although developers could build a variety of interactions into their VR-based loading scenarios, like a balloon machine or a virtual dog [23], they are limited by the low computing power due to the loading process. Whether one should prioritize shorter loading times over less interactivity in a VR-based context is unclear.

Higher immersion can also intensify unpleasant experiences, like fear in horror games [18]. Especially negative emotions, like fear and anxiety [10], have been found to be stronger amplified by immersion than other emotions, like happiness [5]. In the context of VR-based loading screens, possible negative emotions evoked by the forced break could be intensified compared to a less immersive representation. 


\section{Hypotheses}

As demonstrated above, perceived LSE is tested for various types of loading screens such as animations, progress bars and interactive screens. However, the question still remains about whether those effects are valid in a VR-based context. As VR technology offers a high immersive environment, loading scenarios might be perceived differently compared to mobile or desktop waiting time scenarios. Therefore, the main goal of our study is to address this question by investigating the effect of two types of loading screens (interactive and passive) in VR on users' LSE for two waiting times (short and long). We assume that the results of Hohenstein [7] on short waiting times also apply in a VR-based context.

H1: During short waiting times in VR, peoples' LSE is better for interactive loading screens than passive loading screens.

Moreover, we assume that precisely because people look for alternative activities during long waiting times in the real world, interactive loading screens lead to higher LSE in a virtual environment, as they offer users possibilities to interact with their environment.

H2: During long waiting times in VR, peoples' LSE is better for interactive loading screens than passive loading screens.

\section{System Description}

To test our hypotheses, we implemented two loading screens. Based on the work of Hohenstein et al. [7] on desktop-based interactive and passive loading screens, we chose a passive animated Newton's cradle and an interactive Newton's cradle. We implemented both loading screens into the existing visualization tool IslandViz [14]. This open-source tool visualizes OSGi-based software projects as islands on a virtual table in VR. On startup, IslandViz is importing the software system from a database and is then generating the island layout and the island shapes at runtime. This loading can take up to several minutes, depending on the size of the software project. During the loading time, the user enters the virtual environment, i.e., puts on the head-mounted display, and waits for the application to finish loading (Figure 1). Then the islands are presented on the virtual table. The loading screens are developed with Unity 2019.1.6f1 using the SteamVR plugin version 2.3.2.

The Newton's cradles - both animated and interactive - consist of five balls each hanging from a thread, attached to the Loading lettering. In the passive version, the user cannot interact with the balls (Figure 1). However, the first ball is swinging automatically after random time periods (2 -5 seconds) causing a constant movement of the balls. In the interactive version, the user can grab a ball by touching it with the HTC Vive controller and holding the trigger button 


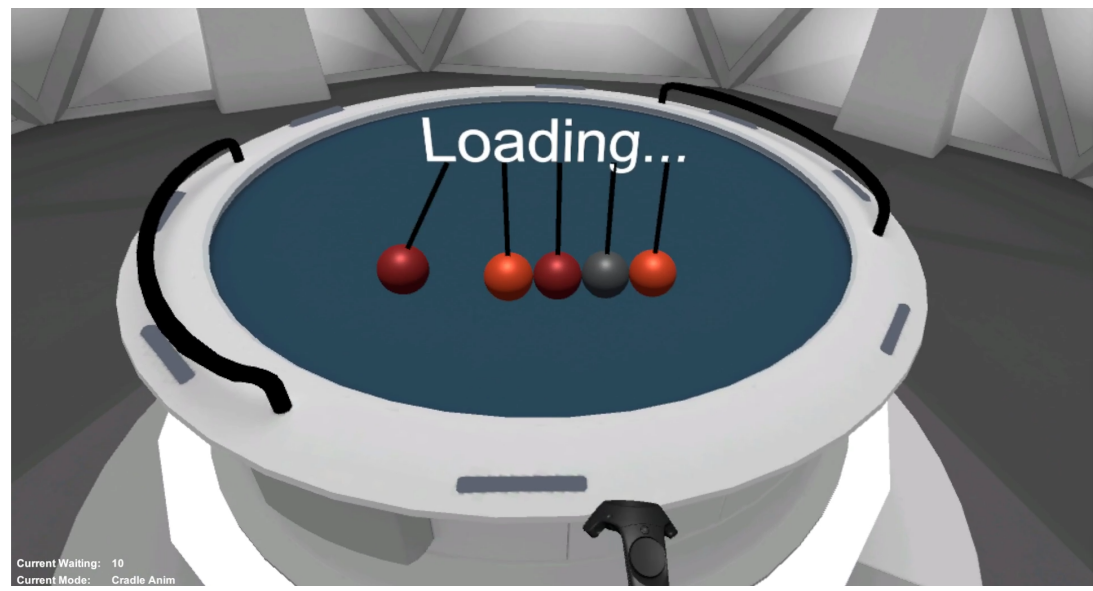

Fig. 1. Passive Loading Screen: The player is waiting in front of the virtual table watching the animated Newton's Cradle with automatically swinging balls.

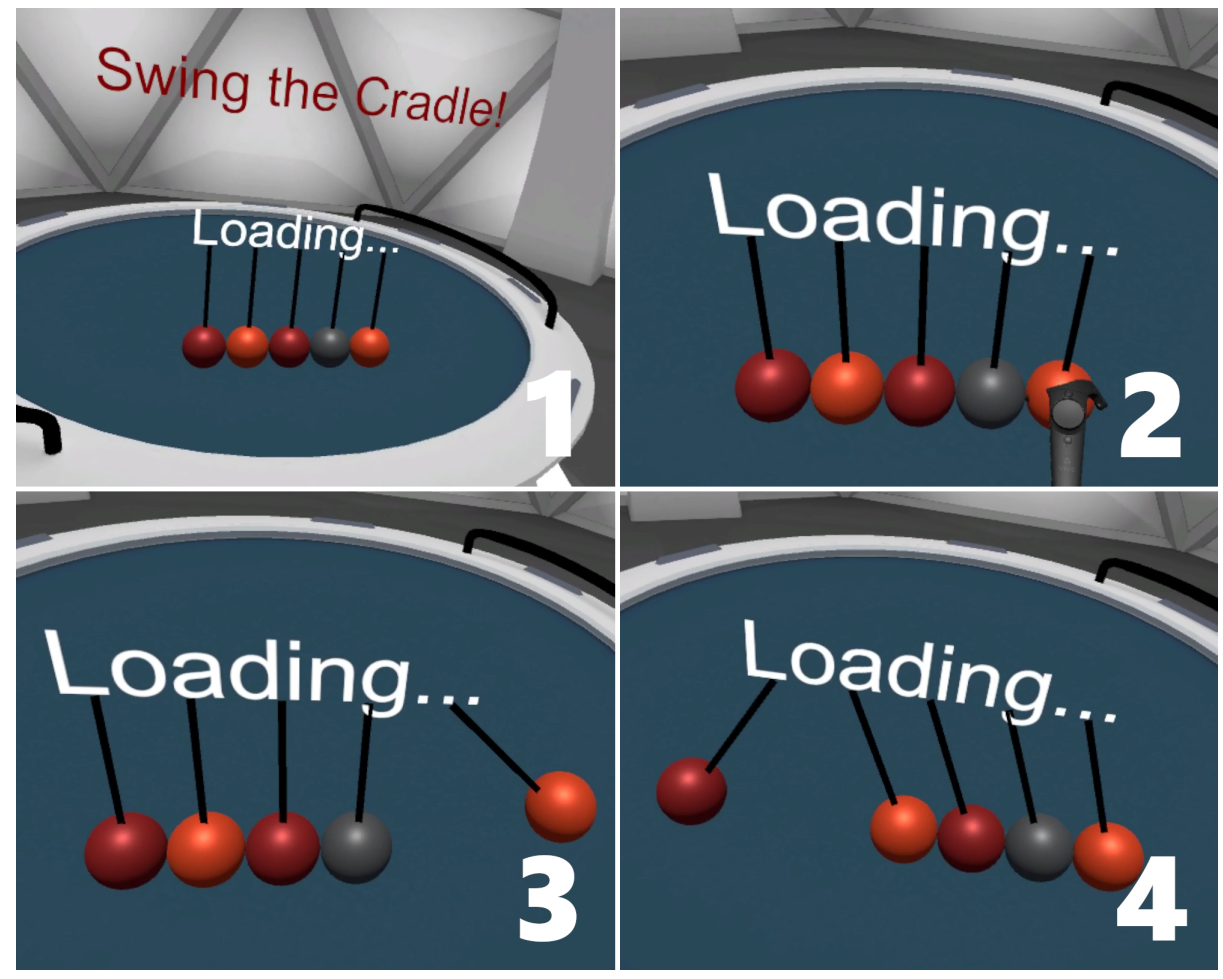

Fig. 2. Interactive Loading Screen: The participant is standing in front of the interactive Newton's cradle (1), touches the right ball (2), grabs and moves the ball (3), and releases the ball to make the whole cradle move (4). 
(Figure 2). The balls interact like a real cradle, i.e., the first ball bumping into the second ball causing the last ball to bounce up and vice versa.

We also manipulated the loading routine of the IslandViz to always take a predefined time. That way, although the loading of the software system did only take a few seconds, the loading screens are always displayed for the time period set by the experimenter. Additionally we added an experimenter interface, so we could change the current loading screen and start a condition, while the participant is immersed. When a loading screen condition is initiated, a Start button appears over the table. To start the loading screen, the user has to press the button, i.e., touch the button with the HTC Vive controller and press the trigger button. That way, the user is always located at the same position, when the loading condition is starting.

\section{Method}

The study employed a $2 \times 2$ within-subjects repeated-measures design. The two independent variables were loading screen (interactive and passive) and waiting duration (10 seconds and 60 seconds). The dependent variables were perceived speed, enjoyment and frustration.

The experiment consisted of two sessions: in the first session, participants experienced both loading screens for 10 seconds each. In the second session, participants experienced both loading screens for 60 seconds each. Thereby we wanted to find out if there are differences concerning users' LSE during long and short waiting times. In addition, we argue that 10 seconds waits in VR might be too fast to perceive the whole environment, especially the interactive loading scenario.

\section{$5.1 \quad$ Measures}

We assessed participants demographic data and their VR experience as a possible influencing variate. Perceived speed, enjoyment and frustration were measured on a 7-point Likert scale: 'How fast did you experience loading the application?' $(1=$ very slow $-7=$ very fast $)$,'How much fun did you have loading the application?' $(1=$ very much $-7=$ not at all $)$, 'How frustrated were you while you were waiting?' $(1=$ not at all $-7=$ very much $)$. Participants had to provide information about those three items every time after they had seen a loading screen. Since we have two waiting times, there are two measures of each perceived speed, enjoyment, and frustration for every loading screen. In the end of the study, we asked participants to rank both loading screens according to their preference.

\subsection{Procedure}

All questionnaires and experimental stimuli were administered to the participants in a laboratory setting. For the experiment, we used the HTC Vive VR 
system, consisting of the head-mounted display, two controllers and two base stations which were installed in our VR lab. Participants had enough space to move in the VR environment. The whole examination including instructions and debriefing took approximately 20 minutes.

The part of the study that took place in VR was about 10 minutes on average and consisted of two sessions, respectively 10 and 60 seconds. The order of the loading screens within the first session was randomized for each participant and remained the same for the second session as well.

After each loading screen, participants were asked to rank their experience about their perceived speed, enjoyment and frustration. Each question was displayed within the virtual environment while the participants had to answer verbally. After experiencing the screens in both sessions, participants were asked to rank the two loading screens according to their preference. The study was ended by open questions and the opportunity to share feedback, concerns or ideas regarding this evaluation.

\subsection{Participants}

In total, 25 persons (12 female, 13 male) participated in our study with a mean age of $28.6(S D=5.58)$. The participation was voluntary and participants were recruited through a local call for participation. All participants have a background in software engineering and have already worked with this tool, i.e., all participants know why the loading time exists (depending on the size of the visualized software project). 3 (12\%) participants stated to have no VR experience. $14(56 \%)$ participants stated to have little VR experience, and $6(24 \%)$ stated to have some experience. $2(8 \%)$ participants stated to use VR technology in their daily working life.

\section{Results}

We predicted that being confronted with an interactive loading screen compared to being confronted with a passive loading screen would improve participants' LSE, thus increase their perceived speed and enjoyment, and decrease their frustration. We performed statistical analysis using the Statistical Package for the Social Sciences (SPSS) Version 22.9 for Windows. For every method, the error probability was set to a significance level alpha $=5 \%$.

\subsection{Perceived Speed}

A repeated measures analysis of variance (ANOVA) with a Greenhouse-Geisser correction determined that mean perceived speed levels showed a statistically significant difference between the interactive and passive loading scenario, $F(1,24)=$ $55.40, p<.001, \eta^{2} p=.70$ (Figure 3). Moreover, there is a significant difference in the mean enjoyment levels between the short and long waiting time, 


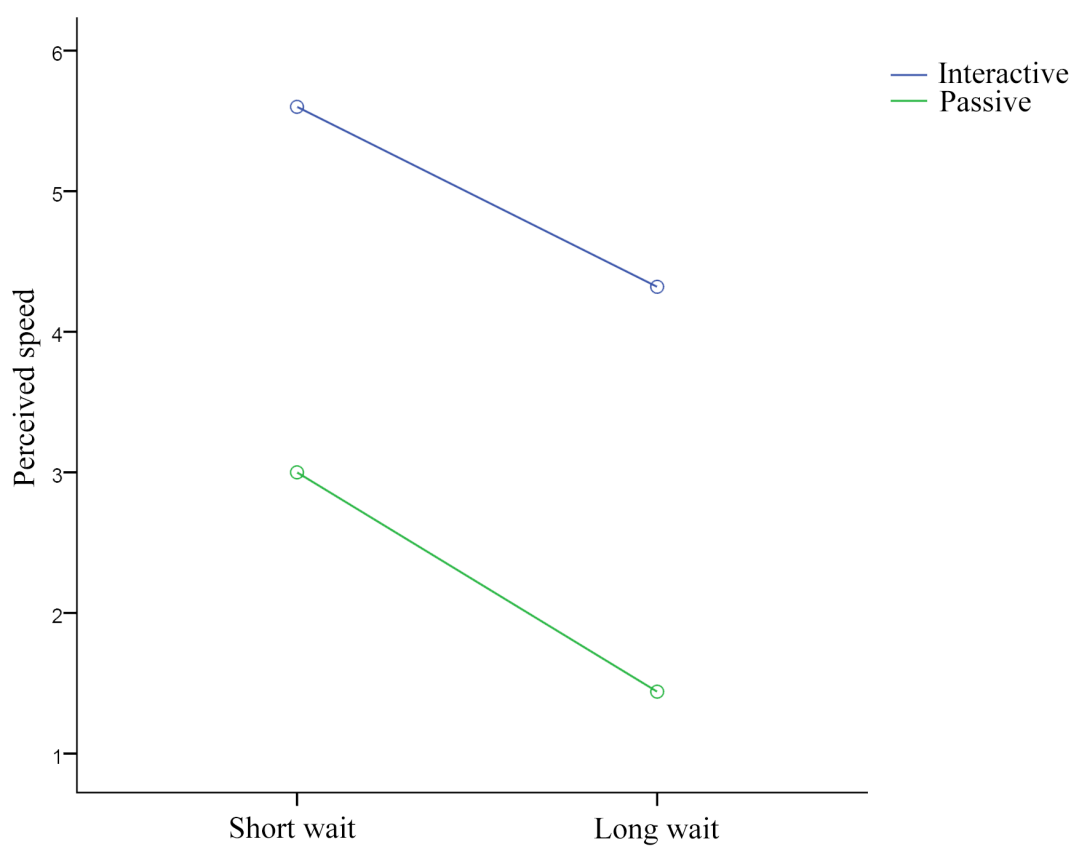

Fig. 3. Effects of interactive and passive loading screens on perceived speed. Note: The vertical axis reflects the degree of perceived speed, higher scores on this measure represent faster perceived loading times. The horizontal axis represents the waiting duration, whereas short wait represents the 10 second wait and long wait represents the 60 second wait.

$F(1,24)=142.73, p<.001, \eta^{2} p=.86$. There is no interaction between loading screen and waiting duration, $p>.05$.

Regardless the waiting time, participants were more likely to perceive waiting times faster when confronted with an interactive loading screen $(M=4.3, S D=$ $.22)$ than with a passive loading screen $(M=2.8, S D=.16)$.

\subsection{Enjoyment}

Another repeated measures ANOVA revealed statistically significant differences in the mean enjoyment levels between the two loading scenarios $(F(1,24)=$ $\left.77.42, p<.001, \eta^{2} p=.76\right)$ and the two waiting times $(F(1,24)=13.54, p=.001$, $\left.\eta^{2} p=.36\right)$ (Figure 4). There is no interaction between loading screen and waiting duration. Regardless the waiting time, participants were more likely to enjoy being confronted with an interactive loading screen $(M=4.5, S D=.24)$ than with a passive loading screen $(M=2.2, S D=.20)$. 


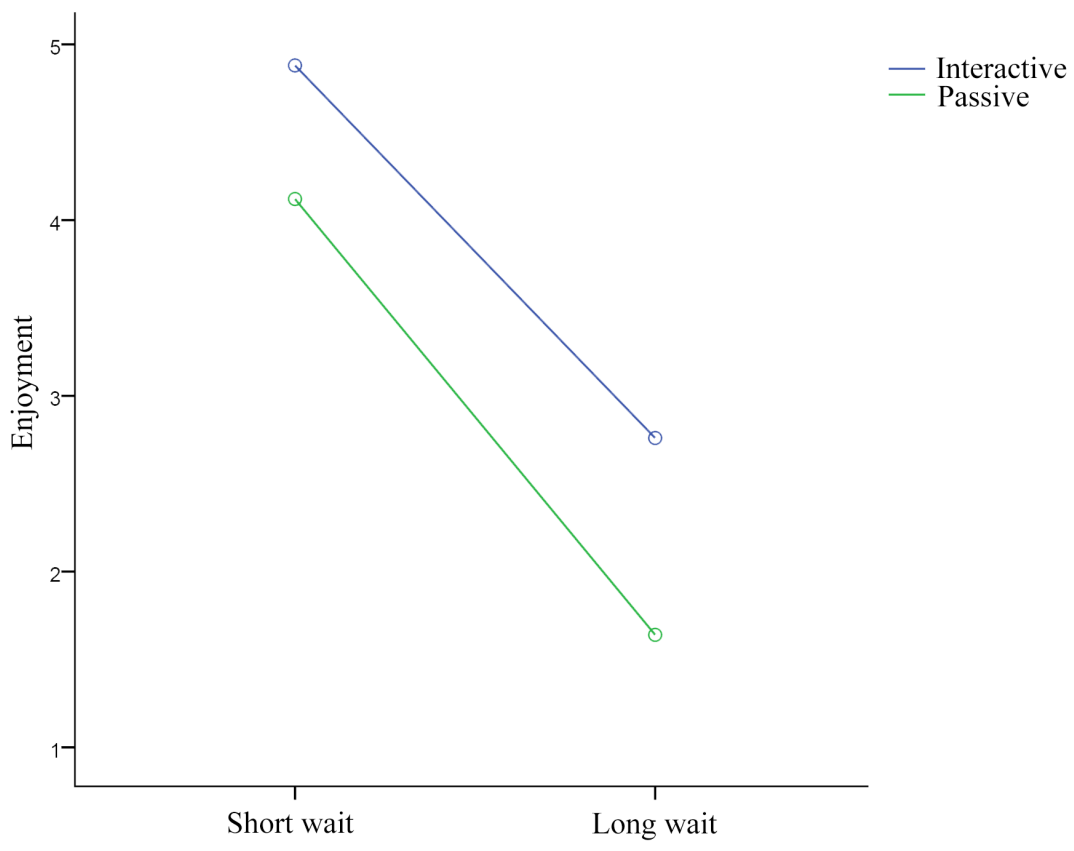

Fig. 4. Effects of interactive and passive loading screens on enjoyment. Note: The vertical axis reflects the degree of enjoyment, higher scores on this measure represent more enjoyment. The horizontal axis represents the waiting duration, whereas short wait represents the 10 second wait and long wait represents the 60 second wait.

\subsection{Frustration}

A final repeated measures ANOVA revealed statistically significant differences in the mean frustration levels between the two loading scenarios $(F(1,24)=42.38$, $p$ i.001, $\left.\eta^{2} p=.64\right)$ and the two waiting times $(F(1,24)=32.11, p=.001$, $\left.\eta^{2} p=.61\right)$ (Figure 5). There is no interaction between loading screen and waiting duration. Regardless the waiting time, participants were more likely frustrated being confronted with a passive loading screen $(M=4.1, S D=.26)$ than with an interactive loading screen $(M=2.5, S D=.22)$.

Overall, the results suggested that participants estimated LSE to be better for the interactive loading condition regardless the waiting duration.

Hence, our hypotheses $\mathrm{H} 1$ and $\mathrm{H} 2$ are confirmed.

\subsection{Preference}

Only one participant (4\%) preferred the animated Newton's Cradle over the interactive Newton's Cradle. 


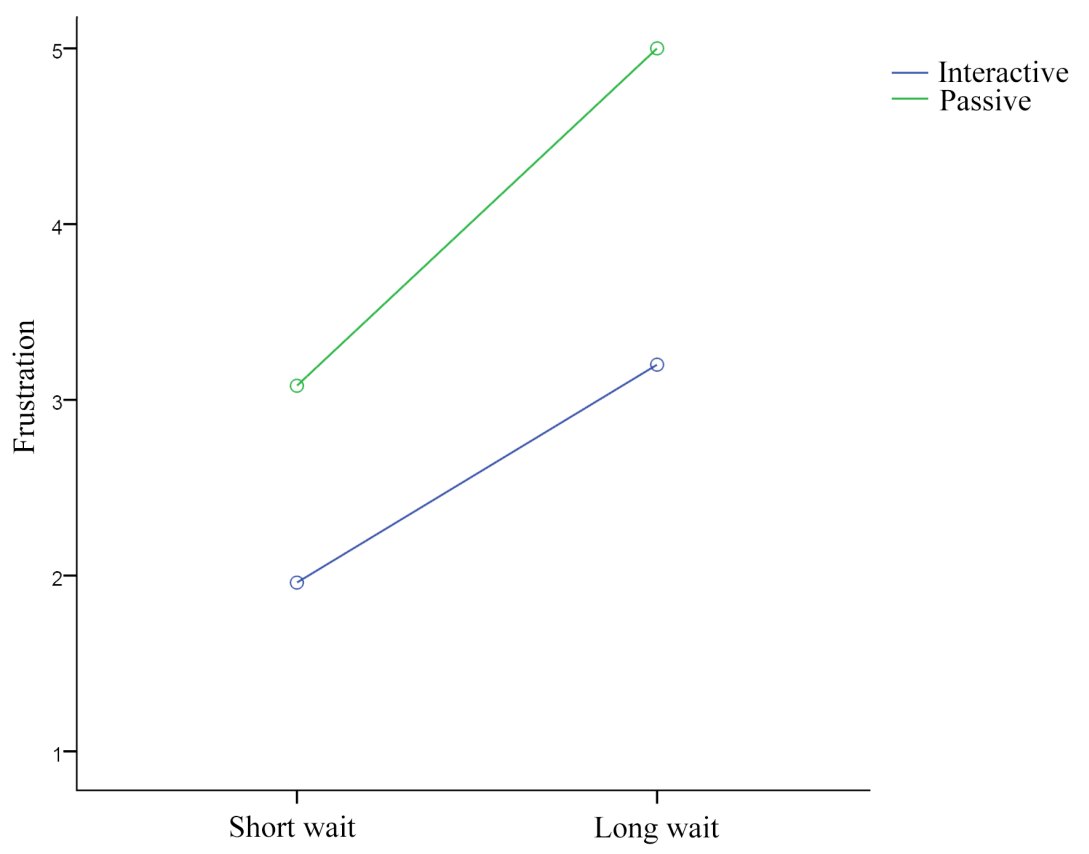

Fig. 5. Effects of interactive and passive loading screens on frustration. Note: The vertical axis reflects the degree of frustration, higher scores on this measure represent more frustration. The horizontal axis represents the waiting duration, whereas short wait represents the 10 second wait and long wait represents the 60 second wait.

\section{Discussion}

Previous research investigated various feedback types that might influence users' loading screen experience (LSE) in a 2D-based environment $[13,8]$. According to previous research users prefer interactive loading screens over passive loading screens [7]. In this study, we investigated interactive and passive loading screens in a VR-based environment to validate those results for VR-based applications. Additionally, we added a short and a long waiting time condition for each loading screen. Overall, the results suggested that participants estimated LSE to be better for the interactive loading condition regardless the waiting duration. In addition, the participants indicated that they favoured the interactive cradle over the animated cradle. Hence, previous research on 2D-based loading screens seem to be valid for VR-based environments and our hypotheses H1 and H2 are confirmed.

Interestingly, our findings are contradictory with popular VR-based video games, like The Lab [23]. However, it is uncertain whether these loading screens are conscious design decisions or whether these loading screens are predeter- 
mined by the game engines and/or software architecture. Since our findings indicate significant differences between the loading screens, the interactivity of a loading screen could be an important factor to consider for good user experience design. This might be particularly critical for applications with many loading screens and is independent of the waiting duration. Even for short waiting times of a few seconds, interactive loading screens are perceived faster and more enjoyable. Hence, based on our findings, we recommend to include interactive loading screens in VR-based applications to reduce frustration and to increase perceived speed and enjoyment during loading.

\subsection{Limitations}

VR-based applications can be very variable and range from a black void with no embodiment at all to a photo-realistic environment with full body tracking. We would argue that, in a default VR application, one can at least see the movement of the controllers in the virtual environment. Hence, a loading scenario in VR is never a strictly passive but rather an interactive experience. Technically this means our passive loading scenario really was a less-interactive loading screen. However, this logic could also be applied to a desktop-based loading scenario, where the user's hand is represented as a mouse courser.

Secondly, the repeated measure in our study design might have led to some sort of learning effect in the long waiting time condition. Since experiencing a loading screen multiple times during a play session is a realistic use case and all participants have experienced the same loading screens prior to the long conditions, we believe that comparing the two loading screens within the long wait time condition is still valid. However a conclusion about the difference between the short wait condition and the long wait condition is not possible.

Thirdly, our participants were not interacting with the software visualization tool after the loading. This might have weakened the strength of the measured LSE. However, all participants have used the IslandViz application before and knew what they were waiting for. This probably would have been a much bigger limitation with participants that are familiar with IslandViz.

Fourthly, we tested our loading screens in the context of software visualization, thus in a context of work. A different context where participants might be more motivated to see what comes after the loading screen, e.g., in video games, the measured effects might also be stronger.

Fifthly, our study was conducted with a relatively small sample of only computer scientists which does not cover a broad population. Hence, a future study should definitely have a bigger sample size and cover a broader population.

\subsection{Future Research}

First, we must compare VR-based loading scenarios with 2D-based loading scenarios. This will answer the question whether there is any influence of immersion, i.e., being locked away from the real world, on the LSE. Other possible VR-related factors influencing the LSE could be embodiment and presence. 
Second, it is necessary to test other loading screens in a VR-based environment. Since we did not indicate the remaining waiting time in our study, another loading screen could be progress bars that indicate the remaining waiting time. This is a viable alternative to static loading screens [15]) and, in a desktop-based context, people should prefer a progress bar over a passive Newton's Cradle [7].

\section{Conclusion}

This study is a first step to understand the influence of different loading screens in VR on users' perceived speed, enjoyment and frustration. However, further analysis is necessary for understanding how loading screens can serve as effective time shortening-tools in a virtual environment. As for desktop or mobile applications, interactive loading screens in VR seem to be a generally better choice. Anyway, this study investigated the user's first contact with a loading screen. This raises the question of whether there is at all influence of immersion on the loading screen experience and whether those effects are accurate for repeated interactions.

\section{References}

1. Antonides, G., Verhoef, P.C., van Aalst, M.: Consumer perception and evaluation of waiting time: A field experiment. Journal of Consumer Psychology 12(3), 193$202(2002)$

2. Blaszczynski, A., Cowley, E., Anthony, C., Hinsley, K.: Breaks in play: Do they achieve intended aims? Journal of Gambling Behavior 32(2), 789-800 (2015). https://doi.org/10.1007/s10899-015-9565-7

3. Bouch, A., Kuchinsky, A., Bhatti, N.T.: Quality is in the eye of the beholder: Meeting users' requirements for internet quality of service. In: Proceedings of the SIGCHI conference on Human Factors in Computing Systems (CHI '00). pp. 297304 (2000)

4. Ceaparu, I., Lazar, J., Bessiere, K., Robinson, J., Shneiderman, B.: Determining causes and severity of end-user frustration. International Journal of Human-Computer Interaction 17(3), 333-356 (2003). https://doi.org/10.1207/s15327590ijhc1703_3

5. Freeman, J., Lessiter, J., Pugh, K., Keogh, E.: When presence and emotion are related, and when they are not. In: Proceedings of the 8th Annual International Workshop on Presence (PRESENCE 2005). pp. 213-219. International Society for Presence Research (2005)

6. Gilleade, K.M., Dix, A.: Using frustration in the design of adaptive videogames. In: Proceedings of the 2004 ACM SIGCHI International Conference on Advances in Computer Entertainment Technology. pp. 228-232. ACM (2004)

7. Hohenstein, J., Khan, H., Canfield, K., Tung, S., Perez Cano, R.: Shorter wait times: The effects of various loading screens on perceived performance. In: Proceedings of the 2016 CHI Conference Extended Abstracts on Human Factors in Computing Systems. pp. 3084-3090. ACM (2016)

8. Hui, M.K., Tse, D.K.: What to tell consumers in waits of different lengths: An integrative model of service evaluation. Journal of Marketing 60(2), 81-90 (1996). https://doi.org/10.2307/1251932 
9. Hurter, C., Girouard, A., Riche, N., Plaisant, C.: Active progress bars: Facilitating the switch to temporary activities. In: CHI'11 Extended Abstracts on Human Factors in Computing Systems. pp. 1963-1968. ACM (2011)

10. IJsselsteijn, W., Ridder, H.d., Freeman, J., Avons, S.E., Bouwhuis, D.: Effects of stereoscopic presentation, image motion, and screen size on subjective and objective corroborative measures of presence. Presence: Teleoperators \& Virtual Environments 10(3), 298-311 (2001). https://doi.org/10.1162/105474601300343621

11. Kim, W., Xiong, S., Liang, Z.: Effect of loading symbol of online video on perception of waiting time. International Journal of Human-Computer Interaction 33(12), 1001-1009 (2017). https://doi.org/10.1080/10447318.2017.1305051

12. Lallemand, C., Gronier, G.: Enhancing user experience during waiting time in hci: Contributions of cognitive psychology. In: Proceedings of the Designing Interactive Systems Conference. pp. 751-760. ACM (2012)

13. Li, S., Chen, C.H.: The effects of visual feedback designs on long wait time of mobile application user interface. Interacting with Computers 31(1), 1-12 (March 2019)

14. Misiak, M., Schreiber, A., Fuhrmann, A., Zur, S., Seider, D., Nafeie, L.: Islandviz: A tool for visualizing modular software systems in virtual reality. In: 2018 IEEE Working Conference on Software Visualization (VISSOFT). pp. 112-116 (Sep 2018). https://doi.org/10.1109/VISSOFT.2018.00020

15. Myers, B.A.: The importance of percent-done progress indicators for computerhuman interfaces. In: Proceedings of the SIGCHI conference on Human Factors in Computing Systems (CHI '85). pp. 11-17 (1985)

16. Nielsen, J.: Usability engineering. Elsevier (1994)

17. Pallavicini, F., Cipresso, P., Raspelli, S., Grassi, A., Serino, S., Vigna, C., Triberti, S., Villamira, M., Gaggioli, A., Riva, G.: Is virtual reality always an effective stressors for exposure treatments? some insights from a controlled trial. BMC psychiatry 13(1), 52 (2013). https://doi.org/10.1186/1471-244X-13-52

18. Pallavicini, F., Ferrari, A., Pepe, A., Garcea, G., Zanacchi, A., Mantovani, F.: Effectiveness of virtual reality survival horror games for the emotional elicitation: Preliminary insights using resident evil 7: Biohazard. In: International Conference on Universal Access in Human-Computer Interaction. pp. 87-101. Springer (2018). https://doi.org/10.1007/978-3-319-92052-8_8

19. Pausch, R., Crea, T., Conway, M.: A literature survey for virtual environments: Military flight simulator visual systems and simulator sickness. Presence: Teleoperators \& Virtual Environments 1(3), 344-363 (1992)

20. Romano, S., Capece, N., Erra, U., Scanniello, G., Lanza, M.: On the use of virtual reality in software visualization: The case of the city metaphor. Information and Software Technology (2019). https://doi.org/10.1016/j.infsof.2019.06.007

21. Slater, M., Wilbur, S.: A framework for immersive virtual environments (five): Speculations on the role of presence in virtual environments. Presence: Teleoperators \& Virtual Environments 6(6), 603-616 (1997). https://doi.org/10.1162/pres.1997.6.6.603

22. Söderström, U., Bååth, M., Mejtoft, T.: The users' time perception: The effect of various animation speeds on loading screens. In: Proceedings of the 36th European Conference on Cognitive Ergonomics. p. 21. ACM (2018)

23. Valve: The lab. https://store.steampowered.com/app/450390/TheLab/ (2016)

24. Zhao, W., Ge, Y., Qu, W., Zhang, K., Sun, X.: The duration perception of loading applications in smartphone: Effects of different loading types. Applied Ergonomics 65, 223-232 (2017). https://doi.org/10.1016/j.apergo.2017.06.015 